\title{
Off-Vertical Axis Rotational Assessment of Transdermal Scopolamine for Motion Sickness Prophylaxis
}

\author{
Daniel Woodard ${ }^{1}$, Glenn Knox $^{2}$, Scott Wood ${ }^{3}$, and Cathy DiBiase ${ }^{1}$ \\ ${ }^{1}$ Bionetics Corporation, NASA-Kennedy Space Center, FL; ${ }^{2}$ University of Florida College of Medicine, UF Health- \\ Jacksonville, Jacksonville, FL; ${ }^{3}$ Department of Neurotology Research, Legacy Health System, Portland, OR
}

\begin{abstract}
The objectives of this study were to evaluate transdermal scopolamine for motion sickness prophylaxis, and to evaluate off-vertical axis rotation (OVAR) as a laboratory model of motion sickness. This was a randomized, prospective, double-blind study design, set in a vestibular research laboratory. The experimental subjects consisted of 12 patients -7 male, 5 female ages 21 to 57, with normal auditory/vestibular function. The intervention was off-vertical axis rotation 20 degrees in the dark after administration of transdermal scopolamine or placebo. The main outcome measures were time duration of tolerated off-vertical rotation, and subjective symptom reporting during rotation at one-minute intervals on a 0-4 scale. Results were as follows: patients treated with transdermal scopolamine had statistically significant improved tolerance time to off-vertical axis rotation. Reported symptom-atology on the $0-4$ subjective
\end{abstract}

Key words: Off-Vertical; Vestibular; Motion Sickness; Scopolamine; Rotational; OffVertical Rotation; OVAR

Correspondence to: Glenn W. Knox, MD University of Florida Health - Jacksonville Department of Surgery 653 West Eighth Street Jacksonville, FL 32209

Telephone: 904-244-3498

E-mail: gwknox@bellsouth.net symptom scale was significantly improved, as compared to placebo, and was dose-dependent. Conclusions are as follows: off-vertical axis rotation is a useful modality for the evaluation of motion sickness medications. Transdermal scopolamine showed statistically significant dosedependent effects in mitigating OVAR-induced motion sickness symptomatology and was well tolerated.

\section{INTRODUCTION}

Motion sickness research has been characterized in the past by exposing experimental subjects to noxious motion stimuli. Then, various qualitative and semi-quantitative measures would be used to evaluate the efficacy of various pharmacologic and non-pharmacologic countermeasures. A convenient, easily reproducible laboratory method of generating motion sickness would greatly facilitate research in this area. In the past, various rotational chair regimes have been utilized for motion sickness research. The present study utilizes off-vertical axis rotation, which efficiently and reliably generates motion sickness symptoms in a laboratory setting. Offvertical axis rotation (OVAR) provides a constantly changing gravitational vector (Wood, 2002). OVAR has been used extensively in the quantitative evaluation of nystagmus and otolith function (Oman, 1982). The continuously changing input to the vestibular system during OVAR may be responsible for the induction of motion sickness. In the present study, OVAR was 
used to measure the efficacy of transdermal scopolamine for motion sickness prophylaxis. Transdermal scopolamine is a prototypical motion sickness medication which is widely known and used (Nachum et al., 2006), thus it was selected as the first pharmacologic countermeasure to be evaluated using off-vertical axis rotation.

This study evaluated the effects of standard and half-dose transdermal scopolamine in preventing motion sickness induced by OVAR. Our hypothesis is that subjects who have undesirable levels of side effects with the full dose will have fewer side effects and will still have effective protection from motion sickness with the lower dose.

The protocol is specifically oriented toward the problem of identifying medications that can be used in an operational setting without inducing side effects. To accomplish this, a subjective scale was used to record both side effects and effects of nausea, before, during, and after exposure to a 15-minute constant-intensity provocative stimulus.

This study was suggested by NASA-Kennedy Space Center (KSC) personnel who are exposed to motion sickness and who report good results from using the scopolamine patch cut in half. However, they are concerned that there is no medical guidance as to whether this is an acceptable procedure. This project may allow us to identify promising alternatives to existing motion sickness medications in improving human performance in provocative environments at KSC.

\section{MATERIALS AND METHODS}

Institutional Review Board (IRB) approval for the study and the use of human subjects was obtained from the KSC IRB. Selection of research subjects was from the KSC medical subject pool and KSC employee volunteers. There were 12 subjects -7 male, 5 female - ages 21 to 57, with normal auditory/vestibular function. Vestibular function was assessed using electronystag-mography (ENG). This test consists of carefully measuring involuntary eye movement (nystagmus) while the subject's balance system is stimulated in different ways. There are four main parts to the ENG: the calibration test, the tracking test, the positional test, and the caloric test. The calibration test evaluates rapid eye movements.
The tracking test evaluates movement of the eyes as they follow visual target. The positional test measures dizziness associated with positions of the head. The caloric test measures responses to warm and cold water or air. Normative values are applied for all four tests. Auditory function was assessed using behavioral audiometry. Normal auditory function is considered to be less than 20 $\mathrm{dB}$ decrement in acuity at $500 \mathrm{~Hz}, 1000 \mathrm{~Hz}, 1500$ $\mathrm{Hz}$, and $2000 \mathrm{~Hz}$.

\section{Medications}

Standard transdermal scopolamine patches ("Transderm-Scop" (Novartis)), which contain 1.5 mg of scopolamine, and inactive placebo patches were employed (Gilman, 1990). Subjects had one full and one-half patch in each trial; the actual total amount of scopolamine applied was either 0 (none), $0.75 \mathrm{mg}$, or $1.5 \mathrm{mg}$.

The half patch contains approximately 0.75 mg of scopolamine. Placebo patches were indistinguishable from the actual medication patches. Of course, the half patch placebo and the full patch placebo had the equal amount of zero scopolamine.

\section{Subjective Reporting Scale}

Subjects verbally reported their condition to the operator at one minute intervals using a subjective reporting scale ranging from $\mathbf{0}$ to $\mathbf{4}$, with the following definitions:

0: No abnormal sensations.

1: Abnormal sensations that do not cause noticeable discomfort, such as dizziness, unusual perspiration, and mild stomach awareness.

2: $\quad$ Mild discomfort, which is noticeable, but is not distracting and would not interfere, even with the performance of demanding tasks.

3: Moderate discomfort, which can be tolerated indefinitely, but is distracting and might affect one's ability to perform demanding tasks.

4: Severe discomfort, which cannot be tolerated indefinitely, or the feeling of impending vomiting. 


\section{Physiologic Testing}

This study required that the subject experience OVAR of sufficient intensity to produce symptoms of motion sickness. To accomplish this, the OVAR chair known as the "JSC Rotator” (depicted in Figure 1) was used.

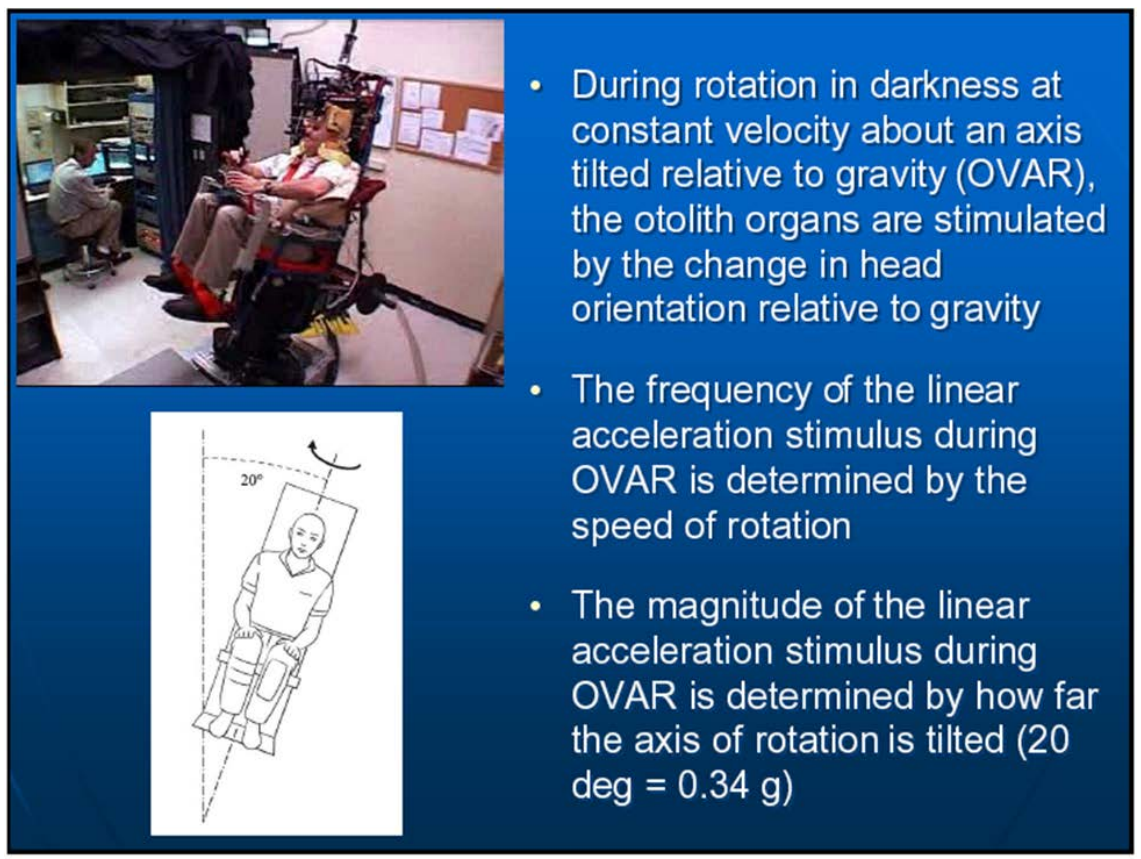

Figure 1. JSC rotator with subject; schematic of OVAR angle.

Each subject participated in three trials each at intervals of at least one week. Each participant was allotted a package of three patches, the full patch, the half patch, and a full placebo patch. Each patch had a serial number for identification and un-blinding at the conclusion of the experiments. After the assignment of the patches, the operator randomly selected one patch at a time for each trial, so the subject and the operator were blinded as to whether drug or placebo was used. All 12 subjects underwent three trials, each trial with a different patch. The procedure was as follows:

1. The subjects applied the patch postauricular 24 hours prior to the exposure.

2. The subjects were seated in the rotating chair and secured with a seat belt, harness, and head restraint.

3. The subjects were rotated at $15 \mathrm{rpm}$ at an angle of 20 degrees off-vertical in low illumination. Subjects wore blacked-out goggles and eyes were closed to eliminate visual motion cues.

4. Rotation was stopped if the subject asked to stop, or if he/she experienced symptoms of Level 4, or when 15 minutes had elapsed-whichever occurred first.

5. Subjective symptoms were recorded at one minute intervals by verbal reporting to the operator.

The JSC Rotator has a head restraint system with moldable pads and adjustable neck and side supports with adequate adjustments to ensure the appropriate subject fit. Straps and padding were designed to restrain the subject securely to the chair, while minimizing pressure points. The restraints can be quickly released, and operators assisted the subjects during ingress and egress from the chair. Each test was monitored by a test conductor, who also recorded data, and a medical monitor (physician or nurse).

This experiment induced at least some symptoms of motion sickness in the majority of 
the subjects. However, the subjects were asked repeatedly to report their level of discomfort and the test was stopped immediately if the subject requested the test be stopped or reported or showed signs of excessive discomfort. The subjects were carefully briefed to assure they understand that the test should be terminated if they experienced Level 4 discomfort, or any other symptoms that they could not tolerate for a prolonged period.

\section{Test Termination Criteria}

There were six criteria established for terminating the test:

1. Subject request, at any time.

2. Subject reports severe (Level 4) or rapidly increasing nausea, or feels that vomiting will occur.

3. Subject reports other discomfort that cannot be easily resolved (i.e., restraint pressure points) or any medical symptoms unrelated to motion sickness, such as shortness of breath or chest pain.

4. Subject demonstrates physical signs of significant motion sickness, or of significant medication side effects.

5. Any unanticipated condition that compromises subject safety or indicates a significant failure in the equipment.

6. Inability of the test monitors to observe and/or communicate with the test subject.
Subjects were evaluated by a physician to exclude any significant medical condition that might make participation unsafe or interfere with data collection. A medical history was completed on each subject to include motion sickness susceptibility and any reactions or allergies to medications. Subjects with a history or family history of glaucoma, or with a history of allergic reaction or side effects to scopolamine, were excluded. If a subject reached Level 4, exposure was stopped and the recovery period was immediately started.

\section{RESULTS}

Although the subjects varied in susceptibility, OVAR reliably induced symptoms of motion sickness, which reached a consistent steady state values during the 15 minute exposure in most subjects. None of the subjects terminated the experiment pre-maturely - i.e., prior to completing the 15 minute exposure. The symptom levels were consistently highest in the placebo groups, lowest in the group given the full $1.5 \mathrm{mg}$ patch, and intermediate in level in the half patch $0.75 \mathrm{mg}$ dosage group (Figure 2). These results were significant to the 0.05 confidence level. Medication side effects were likewise consistently lower in the group receiving the $0.75 \mathrm{mg}$ dosage. Postexposure symptoms of nausea and fatigue were, on the average, more severe in the placebo group.

\section{Motion Sickness Levels During OVAR}

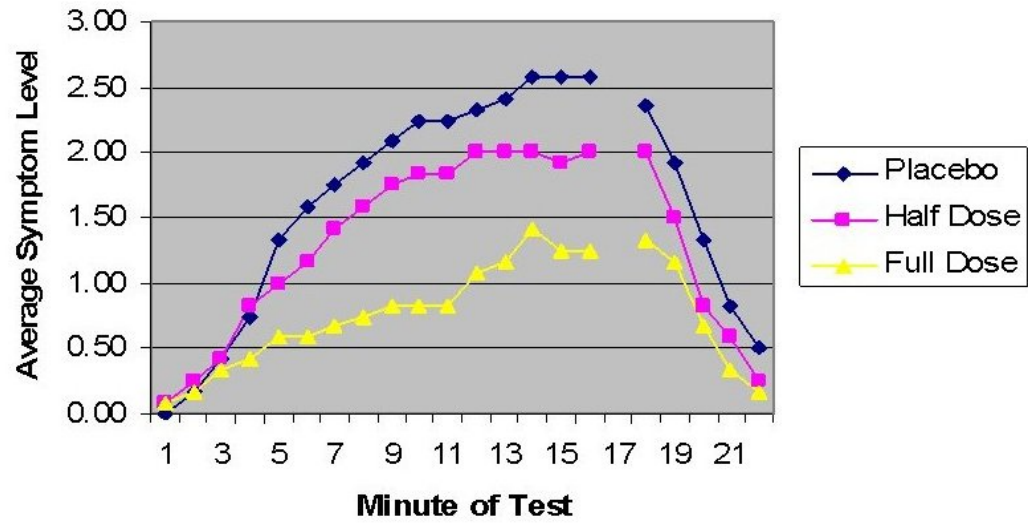

Figure 2. Average symptom score (y-axis) versus elapsed time (x-axis). Series 1 is placebo, Series 2 is the 0.75 mg dose, and Series 3 is the $1.5 \mathrm{mg}$ dose. Data was not collected at minute 17 since subjects egressed the chair at that time period. Data after minute 17 represents continued reporting of symptoms after the rotational stimulus was stopped. Averages of the 0-4 scale can, of course, yield non-integer values. These results were significant to the $\mathbf{0 . 0 5}$ confidence level. 


\section{Statistical Tests}

The symptom scale is nonlinear; consequently, in most cases we used the Friedman analysis of variance test, which requires a block design, but is nonparametric and permits multigroup comparisons. For block designs, there are only a few factors or variables that are of primary interest. However, there are also several other nuisance factors. Nuisance factors are those that may affect the measured result, but are not of primary interest. In these experiments, the nuisance factors include: the specific operator who prepared the treatment, the time of day the experiment was run, and the room temperature.

\section{Side Effect Levels}

Two of 12 subjects reported side effects with the placebo, 3 of 12 with the $0.75 \mathrm{mg}$ dose, and 9 of 12 with the $1.5 \mathrm{mg}$ dose - three of these at Level 2. The $0.75 \mathrm{mg}$ dose caused significantly fewer side effects than the $1.5 \mathrm{mg}$ dose $(\mathrm{p}=$ 0.004 ), but was not significantly different from the placebo $(\mathrm{p}=0.157)$. Of interest, only 3 subjects reported side effects with the half-dose (Figure 3). Although the motion sickness scale is not continuous, a useful indication of overall results can be gained by examining the variation in average motion sickness levels with time. This is just the arithmetic average of the $0-4$ motion sickness scale as shown above. Of course, the arithmetic average of integers can yield non-integer values. Figure 2 shows the average motion sickness levels for subjects taking each of the three doses. The OVAR stimulus is of constant intensity; however, there is clearly a latency period-regardless of dosage level, symptoms reach a stable level only after about 12 minutes of exposure. This suggests that motion sickness tests in which the stimulus intensity is increased at frequent intervals may be measuring latency, rather than susceptibility. Post-test fatigue is even more difficult to measure as a qualitative symptom. We simply relied on a binary 1 for "yes" and 2 for "no" and took the average. This is obviously very simplistic, but does yield some useful information.

\section{Number of Subjects Reporting Side Efects at Each Dosage Level}

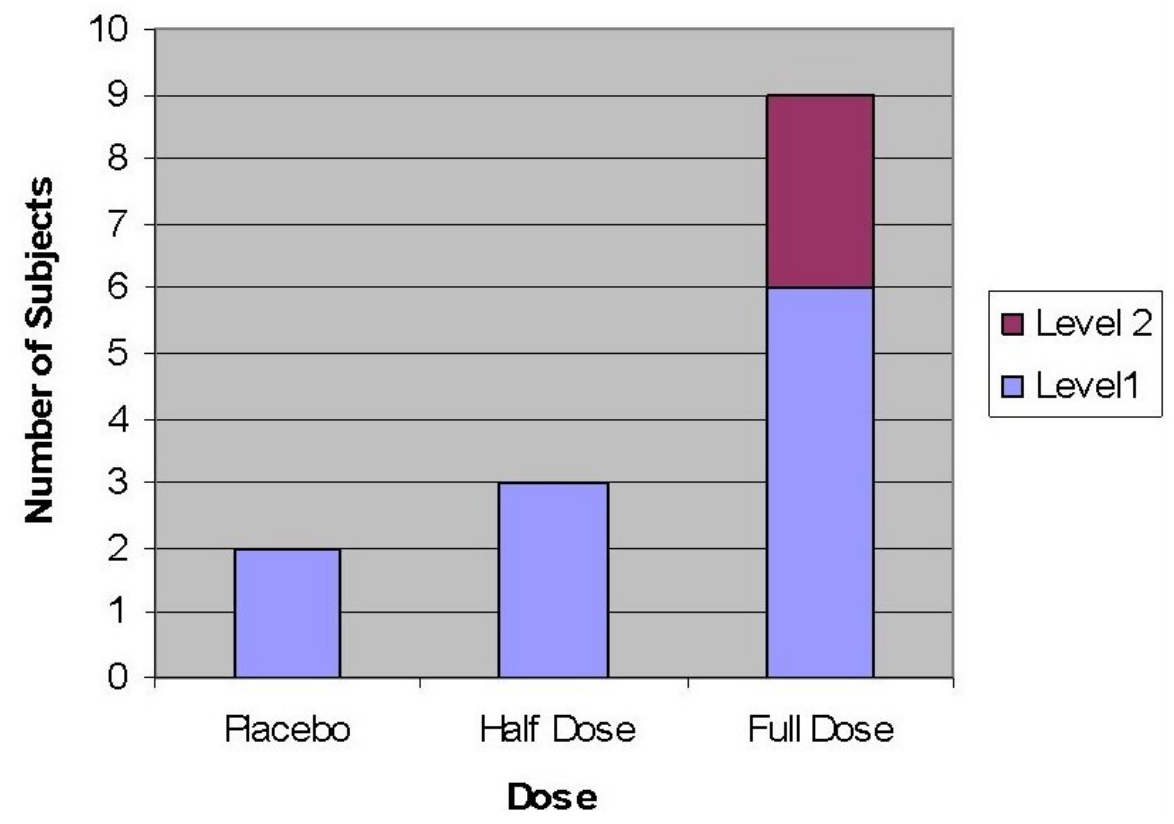

Figure 3. Number of subjects reporting side effects at each dosage level. Note that half-dose side effects were reported by only one more subject than placebo (3 versus 2 ). 


\section{Effect of Dose on Motion Sickness Level}

As expected, the average effect of the halfdose was about half that of the full dose. However, 6 of 12 subjects experienced reduction of maximum symptom level by at least one level with the half-dose, as compared to the full dose. Post-test nausea (Figure 4) and fatigue (Figure 5) were prominent at one and two hours after the exposure, but resolved by three hours. Naïve subjects often experience nausea as the primary symptom of motion sickness. However, subjects who are well-adapted, such as experienced sailors, know fatigue is the most prominent symptom and primary reason for inability to perform occupational tasks. In this test, post-exposure fatigue levels one and two hours after the exposure were significantly higher (Friedman ANOVA, $p=0.011$ ) than those of the medicated subjects. Patients treated for motion sickness with antiemetics after symptoms develop often experience fatigue or drowsiness. Such patients may assume they are medication side effects, but they may instead be symptoms of motion sickness itself. These effects may not occur at all if motion sickness can be prevented with adequate prophylactic medication.

\section{DISCUSSION}

Our initial hypothesis was that subjects who have undesirable levels of side effects with the full dose will have fewer side effects and will still have effective protection from motion sickness with the lower dose. Some subjects received effective protection with the half-dose and thus would benefit from the reduced level of side effects with a lower dose, while others who did not receive complete protection from the $1.5 \mathrm{mg}$ dose but had minimal side effects could theoretically benefit from titrating to a $2.0 \mathrm{mg}$ dose. All motion sickness drugs enter the central nervous system (CNS) and cause dose-related side effects. Therefore, effective treatment must strive to keep the level within a therapeutic range. The difficulty with oral medications is that the level fluctuates each time a dose is taken. Scopolamine has a relatively stable blood and CNS level. Subjects vary considerably in their response to the drug, and with some tolerating larger doses but requiring larger doses for adequate protection, it is desirable to have a method of titrating the dose. Here we have demonstrated that the use of a half patch can be used to titrate to an effective appropriate dose for an optimal level.

\section{Post-test Nausea}

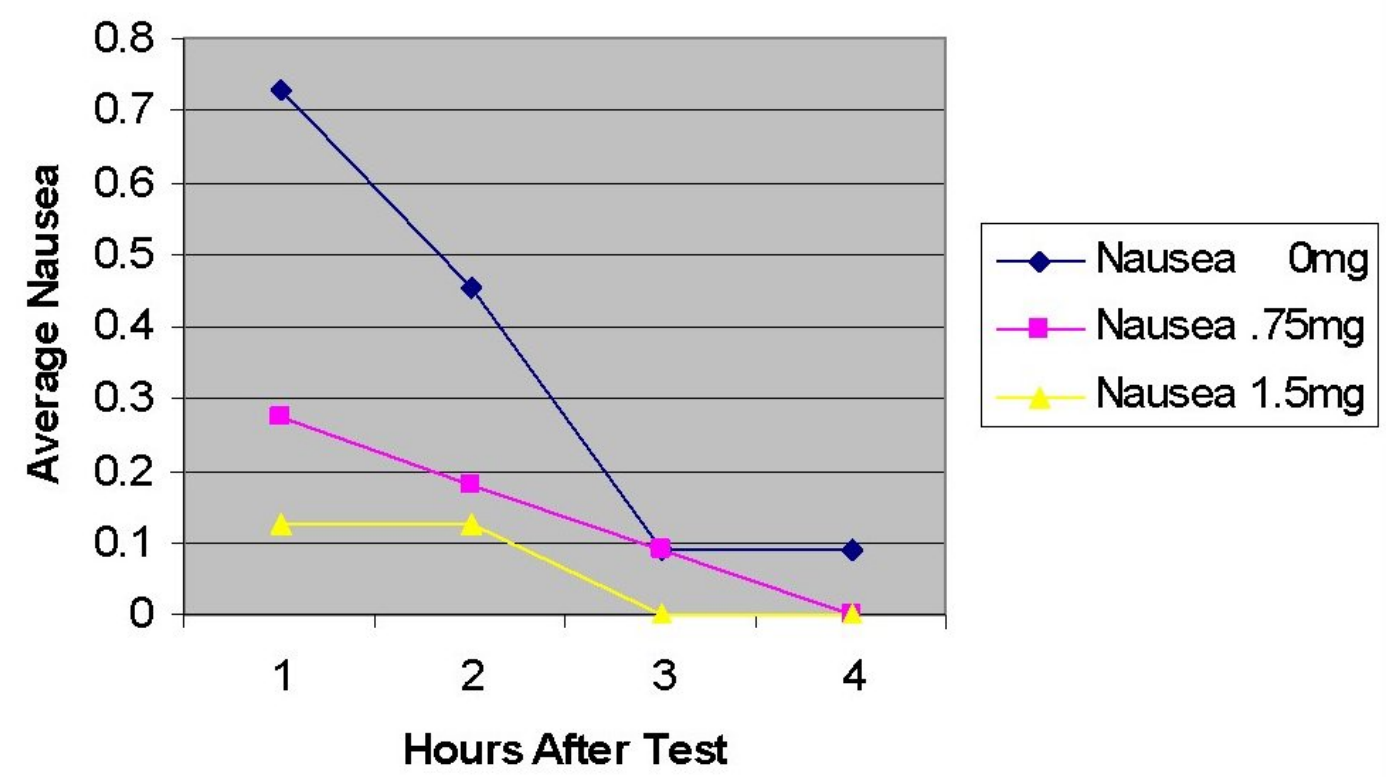

Figure 4. Post-test nausea. 


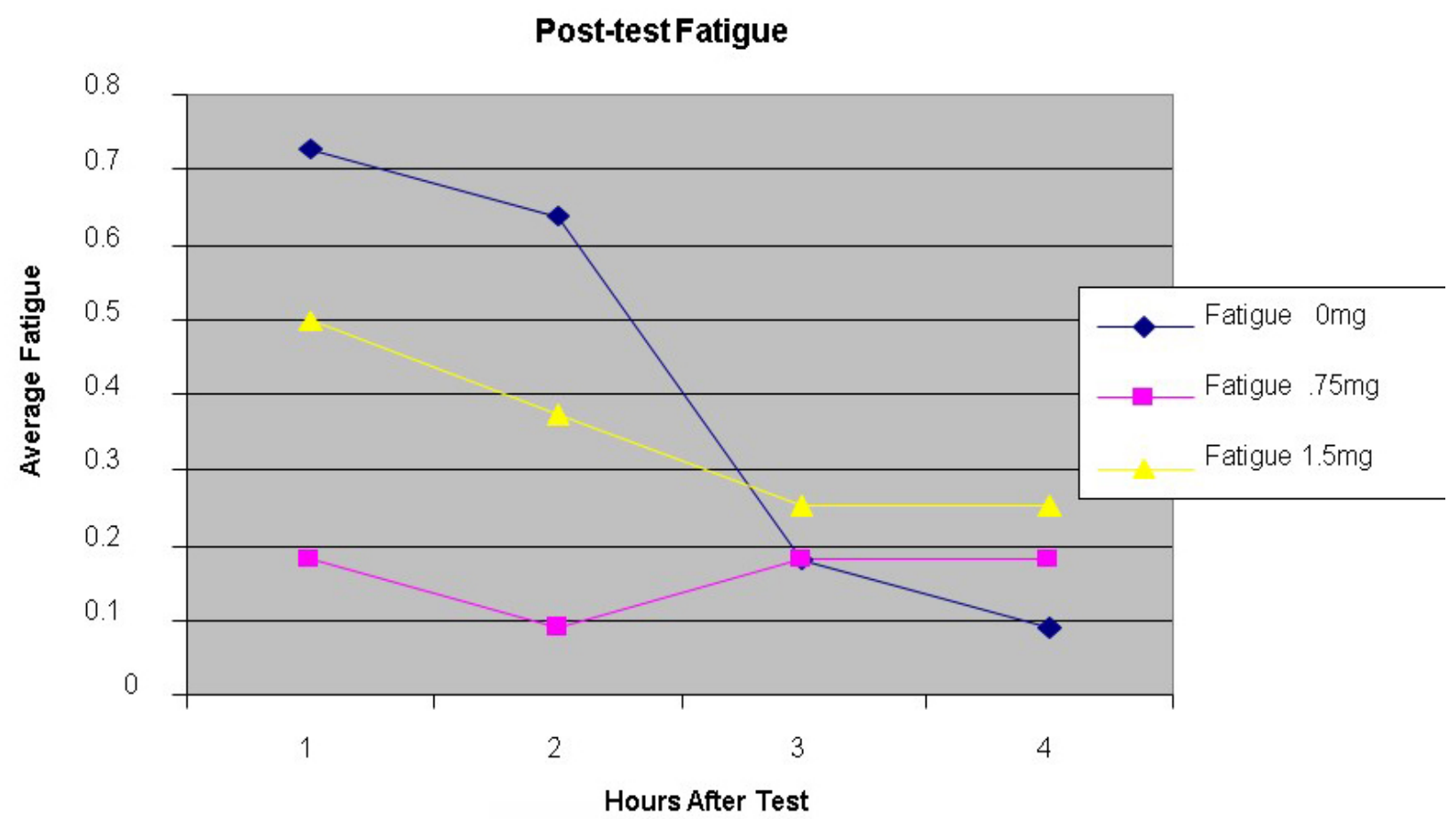

Figure 5. Post-test fatigue.

\section{Effects of Motion Sickness}

Most subjects experienced some motion sickness during the exposure. Motion sickness has been experienced since the earliest days of sea travel and although uncomfortable, it goes away completely without adverse effects. The most common adverse effects are nausea and fatigue. The exposure itself did cause fatigue or drowsiness in some subjects that lasted an hour or more following the test.

\section{Mode of Action and Side Effects of Medications}

Scopolamine blocks the action of the neurotransmitter acetylcholine at muscarinic receptors (Gilman, 1990). It is available by prescription for treatment of motion sickness in two forms: an oral capsule (Scopace), or a patch (Transderm-Scop) (McEvoy, 2005). It is also used by NASA in an oral combination with Dexedrine for motion sickness in space (Davis et al., 1993). Scopolamine commonly causes dry mouth and may cause light sensitivity or slightly blurred vision (Parrott and Jones, 1985). On rare occasions it may cause a temporary confused state similar to alcohol intoxication, or it may exacerbate angle closure glaucoma (Parrott and Jones, 1985).

\section{Limitations to Experimental Design}

The number of subjects was too small to draw any conclusions regarding susceptibility versus age or gender. It has been suggested that genetic factors may play a role in motion sickness susceptibility (Knox, 2014). Further studies would help in elucidating these factors. Another confounding factor is habituation. We tried to mitigate this factor by scheduling each subject's three tests at one week intervals. We also randomly scheduled the three tests vis-à-vis each dosage level. Finally, varying the speed of the rotator in a pseudorandom or random manner would be expected to be much more provocative of motion sickness symptoms.

\section{Other Drugs}

Of course, many other drugs could be tested using this experimental design, such as phenytoin, scop-dex, meclizine, and promethazine, just to name a few. One of the goals of this study was to simply establish OVAR as a valid way to evaluate these drugs in the future. 


\section{CONCLUSIONS}

OVAR is a safe and consistent laboratory model for the generation of motion sickness. This model can be used to evaluate pharmacologic countermeasures for motion sickness. Scopolamine in the transdermal form is effective in the mitigation of motion sickness symptoms, as shown in this experimental model. Scopolamine's mitigating effects against motion sickness appear to be dose-dependent. Further research using OVAR to evaluate other pharmacologic agents, as well as non-pharmacologic methods, to mitigate motion sickness is indicated. All drugs that affect the CNS have side effects. When performance is not critical, motion sickness can often be relieved with a sedating dose of an antiemetic. However, for personnel who must perform critical and hazardous tasks in provocative environments, it is often necessary to find the optimal dose that relieves symptoms without interfering with performance.

\section{ACKNOWLEDGEMENTS}

This study was funded in part by the Aerospace Medicine and Environmental Health Branch, Kennedy Space Center, Florida, and the Florida Space Research Institute.

\section{REFERENCES}

Davis JR, Jennings RT, Beck BG (1993) Comparison of treatment strategies for space motion sickness. Acta Astronautica 29(8): 587-591

Gilman AG (1990) Goodman and Gilman's The Pharmacological Basis of Therapeutics, 8th edn, New York: Pergamon Press

Knox G (2014) Motion sickness: an evolutionary and genetic basis for the negative reinforcement model. Aviation, Space, and Environmental Medicine 85(1): 46-49

McEvoy G (2005) AHFS Drug Information, Bethesda, MD: American Society of Health System Pharmacists, Inc.

Nachum Z, Shupak A, Gordon C (2006) Transdermal scopolamine for prevention of motion sickness: clinical pharmacokinetics and therapeutic applications. Clinical Pharmacokinetics 45: 543-566

Oman C (1982) A heuristic mathematical model for the dynamics of sensory conflict and motion sickness. Acta Otolaryngologica Supplement 392: 1-44

Parrott A, Jones R (1985) Effects of transdermal scopolamine upon psychological test performance at sea. European Journal of Clinical Pharmacology 28: 419-423

Wood S (2002) Human otolith-ocular reflexes during off-vertical axis rotation: effect of frequency on tilt-translation ambiguity and motion sickness. Neuroscience Letters 323: $41-44$ 